OPEN ACCESS

Edited by:

David Rodriguez-Lazaro, University of Burgos, Spain

Reviewed by:

Xuanxian Peng,

Sun Yat-sen University, China

Amjad Ali,

National University of Sciences

and Technology, Pakistan

*Correspondence:

Wenbin Wang

wenbin66@jou.edu.cn

Saikun Pan

pskgx@jou.edu.cn

Specialty section:

This article was submitted to

Food Microbiology,

a section of the journal

Frontiers in Microbiology

Received: 02 November 2020 Accepted: 18 December 2020

Published: 28 January 2021

Citation:

Wang W, Liu J, Guo S, Liu L, Yuan Q, Guo L and Pan S (2021) Identification of Vibrio parahaemolyticus and Vibrio spp. Specific Outer Membrane Proteins by Reverse Vaccinology and Surface

Proteome.

Front. Microbiol. 11:625315 doi: 10.3389/fmicb.2020.625315

\section{Identification of Vibrio}

\section{parahaemolyticus and Vibrio spp. Specific Outer Membrane Proteins by Reverse Vaccinology and Surface Proteome}

\author{
Wenbin Wang1,2,3*, Jianxin Liu'1,2, Shanshan Guo ${ }^{1,2}$, Lei Liu ${ }^{1,2}$, Qianyun Yuan ${ }^{1,2}$, Lei Guo ${ }^{1,2,3}$ \\ and Saikun Pan ${ }^{1,2,3 *}$
}

1 Jiangsu Key Laboratory of Marine Biotechnology, Jiangsu Ocean University, Lianyungang, China, ${ }^{2}$ Co-Innovation Center of Jiangsu Marine Bio-industry Technology, Jiangsu Ocean University, Lianyungang, China, ${ }^{3}$ Jiangsu Key Laboratory of Marine Bioresources and Environment, Jiangsu Ocean University, Lianyungang, China

The discovery of outer membrane proteins (OMPs) with desirable specificity and surface availability is a fundamental challenge to develop accurate immunodiagnostic assay and multivalent vaccine of pathogenic Vibrio species in food and aquaculture. Herein 101 OMPs were systemically screened from 4,831 non-redundant proteins of Vibrio parahaemolyticus by bioinformatical predication of signaling peptides, transmembrane (TM) $\alpha$-helix, and subcellular location. The sequence homology analysis with 32 species of Vibrio spp. and all the non-Vibrio strains revealed that 15 OMPs were conserved in at least 23 Vibrio species, including BamA (VP2310), GspD (VP0133), Tolc (VP0425), OmpK (VP2362), OmpW (VPA0096), LptD (VP0339), Pal (VP1061), flagellar L-ring protein (VP0782), flagellar protein MotY (VP2111), hypothetical protein (VP1713), fimbrial assembly protein (VP2746), VacJ lipoprotein (VP2214), agglutination protein (VP1634), and lipoprotein (VP1267), Chitobiase (VP0755); high adhesion probability of flgH, LptD, OmpK, and OmpW indicated they were potential multivalent Vibrio vaccine candidates. V. parahaemolyticus OMPs were found to share high homology with at least one or two Vibrio species, 19 OMPs including OmpA like protein (VPA073), CsuD (VPA1504), and MtrC (VP1220) were found relatively specific to V. parahaemolyticus. The surface proteomic study by enzymatical shaving the cells showed the capsular polysaccharides most likely limited the protease action, while the glycosidases improved the availability of OMPs to trypsin. The OmpA (VPA1186, VPA0248, VP0764), Omp (VPA0166), OmpU (VP2467), BamA (VP2310), TolC (VP0425), GspD (VP0133), OmpK (VP2362), Ipp (VPA1469), Pal (VP1061), agglutination protein (VP1634), and putative iron (III) compound receptor (VPA1435) have better availability on the cell surface.

Keywords: Vibrio parahaemolyticus, outer membrane protein, identification, surface proteome, bioinformatics, shaving, reverse vaccinology 


\section{HIGHLIGHTS}

- OMPs between Vibrio species had a close and complex genetic relationship.

- The capsule affected surface proteome analysis of V.parahaemolyticus.

- Differential OMPs of V. parahaemolyticus and Vibrio spp. were revealed.

\section{INTRODUCTION}

Vibrio spp. are halophilic bacteria that are widely distributed in seawater, offshore sediments, marine life, and seafood products (Hackbusch et al., 2020). Due to the high prevalence of Vibrio species in seafood, Vibrio-related food poisoning represents the leading cause of infectious diarrhea in coastal regions (Elmahdi et al., 2016). Acute attacks, abdominal pain, and watery symptoms are the main clinical symptoms (Guin et al., 2019). The most prevalent pathogenic Vibrio species in human infections include Vibrio parahaemolyticus, Vibrio vulnificus, and Vibrio cholera, among which V. vulnificus is more life threatening and $V$. parahaemolyticus exhibits a higher prevalence in sea food (Bonnin-Jusserand et al., 2019). Furthermore, the vibriosis caused by Vibrio species (e.g., V. parahaemolyticus, Vibrio anguillarum, Vibrio alginolyticus, Vibrio ordalii, and Vibrio harveyi) has been found to cause an infection in more than 50 economic fish species and is considered a major economic threat to the marine aquaculture industry (Yu et al., 2016).

Rapid diagnostic tests are promising to provide better surveillance in the aquaculture and food industries (Baker-Austin et al., 2018). Although there have been several reports on the preparation of diagnostic antibodies against $V$. parahaemolyticus or Vibrio species by whole cell antigens, flagella, and hemolysins, the specific diagnostic surface antigens are largely unknown (Xu et al., 2019). Also, vaccines are the preferential way to battle vibriosis in aquaculture for sustainability and food safety (Wang et al., 2020). Polyvalent vaccines that cover the main serotypes of the pathogen are preferred when compared with the monovalent vaccine that contains a single strain of a single antigen (Schlingmann et al., 2018). Furthermore, the multiple vaccine (combination vaccine) combined two or more vaccines have effectively protected groupers against multiple Vibrio and viral pathogens (Huang et al., 2012). The traditional strategy of Vibrio polyvalent vaccines is based on the whole-cell antigens of representative strains like $V$. alginolyticus, $V$. parahaemolyticus (Aly et al., 2020). The protection against homologous strains with these vaccines was very effective, but the protection of heterogeneous strains or multiple species were elusive. Outer membrane proteins (OMPs) are currently the main candidate antigens used in polyvalent vaccine studies for their essential function, surface exposure, and conservation among different strains (Pore and Chakrabarti, 2013). There have been some studies of the OmpK and OmpU as polyvalent vaccine candidates (Duperthuy et al., 2010; Li et al., 2010b), while many studies focus on the discovery of more multivalent and potent Omps for future vaccine development.
Based on the bacterial serum-based immunoproteomics, Li et al. (2010a) identified 33 OMPs of $V$. parahaemolyticus on the two-dimensional (2-DE) gel, and found that the OmpA and Pal proteins had a multiple protective effect for the tested strains of Vibrio, Aeromonas, and Pseudomonas in carp. They also revealed the polyvalent vaccines from $V$. alginolyticus by a heterogeneous antiserum-based immunoproteomics with bacterial immunization challenging method (Li et al., 2009). Li et al. (2014) in another group used the same strategy and found 10 immunogenic OMPs, including LptD, and OmpK. Comparative analysis of Omps that respond to fish and human plasma stress by differential sub-proteomic methodologies was also used to develop highly protective vaccine candidates of E. tarda (Wang et al., 2012). DNA shuffling of six OmpA gene from $V$. alginolyticus, $V$. parahaemolyticus, Edwardsiella tarda, and Escherichia coli was also reported to develop polyvalent vaccines against $V$. alginolyticus and $E$. tarda infections (Cheng et al., 2018). However, the multivalence of these proteins among the Vibrio species remains unclear and some conserved OMPs with a lower abundance may be missed due to competition of the immune system for whole-cell immunogen (Shinoy et al., 2013). Another proteomics strategy is the enzymatic shavingbased surface proteome analysis (Rodríguez-Ortega et al., 2006). It was successfully used to identify the surface-exposed proteins of Gram-positive group A Streptococcus, and widely applied to some Gram-negative bacteria (Solis and Cordwell, 2011; OlayaAbril et al., 2014). No shaving-based surface proteome study of Vibrio has been reported.

A genomic and bioinformatic approach, also known as reverse vaccinology, has revolutionized antigen screening and vaccine development by adopting computerized screening of all protein sequences from the target pathogen (Dalsass et al., 2019). Pizza et al. (2000) first reported an analysis of the open reading frames of Neisseria meningitidis and intensive recombinant expression of the 570 potential OMPs (Giuliani et al., 2006). To improve the labor-intensive screening process, many bioinformatics tools have been used to analyze large quantities of genomic or protein sequences (Garcia-Angulo et al., 2014). Specially, to reveal broadspectrum immunogenic targets and analyze the close-related species, the pangenome analysis for the core, accessory, and unique genes was introduced to reverse vaccinology (Zeng et al., 2017; Naz et al., 2019). To our knowledge, the OMPs of $V$. anguillarum have been screened by reverse vaccinology (Baliga et al., 2018). However, little is known about the immunogenic OMPs that are specific to V. parahaemolyticus or Vibrio spp. and represent promising diagnostic antigens and vaccine candidates.

In this study, the OMPs of $V$. parahaemolyticus were comprehensively screened from the 4,831 protein sequences with intergraded bioinformatics predication methods. The differential OMPs and vaccine candidates of $V$. parahaemolyticus and Vibrio spp. were obtained after the overall homology analysis with the other bacteria using the Basic local alignment search tool $\left(\right.$ BLAST, $\left.{ }^{1}\right)$. The possible vaccine candidates were further selected by the adhesion probability analysis. To experimentally study the OMPs of $V$. parahaemolyticus, the surface proteome was analyzed

\footnotetext{
${ }^{1}$ https://blast.ncbi.nlm.nih.gov/Blast.cgi
} 
with enzymatical shaving (glycosidases, trypsin) of the intact cells and mass spectrometry identification of the related peptides.

\section{MATERIALS AND METHODS}

\section{Screening of Signal Peptide-Positive Proteins From the Encoded Proteins of V. parahaemolyticus in the National Center for Biotechnology Information}

The flow chart of OMP screening of $V$. parahaemolyticus is shown in Figure 1.

The 4,831 protein sequences encoded by nucleotides (accession numbers: BA000031.2 and BA000032.2) of the $V$. parahaemolyticus RIMD 2210633 strain were downloaded as FASTA files from the National Center for Biotechnology Information (NCBI) database. The signal peptide is involved in protein transportation and is located on the cell membrane. All of the 4,831 encoded proteins of $V$. parahaemolyticus were analyzed for the presence of the $\mathrm{N}$-terminal signal peptide using SignalP 5.0 (Armenteros et al., 2019). The following parameters were used, and the maximum number of inputted proteins was 5,000 . The organism group was Gram-negative and the output format was a short output.

\section{Predicting the Transmembrane Helices in the Candidate Proteins}

The number of highly hydrophobic TM helices in the proteins was negatively associated with the possibility of recombinant expression. The topology information revealed the location of aminol acids on the protein structure and the cell membrane. The transmembrane (TM) helix and topology of the candidate proteins were predicted using the online server TMHMM 2.0 (Erik et al., 1998) and HMMTOP (also known as Community College Transfer Opportunity Program [CCTOP]) (Tusnády and Simon, 2001). Due to the substantial challenge of multi-pass TM protein expression in the prokaryotic expression system, the proteins with less than two TM helixes were selected for further analysis.

\section{Predicting the Subcellular Localization of the Candidate Proteins}

The bacterial proteins are primarily located in the cytoplasm, the inner membrane (cytoplasmic membrane), periplasm, outer membrane, and extracellularly. The subcellular locations of the obtained proteins with less than two TM helixes were predicted by PSORTb, CELLO, and UniProt. PSORTb is a web-based tool that provides a link to the PSORT family of programs for predicting the subcellular localization (Yu et al., 2010). CELLO based on Support Vector Machine was used to detect specific amino acid compositions and motifs, thereby predicting the subcellular location of proteins (Yu et al., 2004). The UniProt database provides a comprehensive, high-quality, and freely accessible resource of protein information, including the location and topology of the mature proteins in the cell (The UniProt, 2018). To cross-check the results and improve the coverage and accuracy of prediction, proteins predicted as the OMP by more than one method were selected for further analysis.

\section{Sequence Homology Analysis of the Candidate Proteins With Large Number of Strains}

Basic local alignment search tool is an online tool used to identify the local similarity between sequences. This program is supported by the NCBI to compare the nucleotide or protein sequences with sequence databases and calculate the statistical significance of matches. BLAST can also be used to infer the functional and evolutionary relationships between sequences and help identify the protein families. The BLAST program was used to determine the homology with all the identified OMPs among $V$. parahaemolyticus serovars, as well as those present in the other 32 Vibrio spp. (Vibrio cholerae; V. vulnificus; V. alginolyticus; Vibrio diabolicus; Vibrio antiquarius; Vibrio campbelli; V. harveyi; Vibrio owensii; Vibrio rotiferianus; Vibrio jasicida; Vibrio natriegens; $V$. alfacsensis; V. coralliilyticus; V. anguillarum; Vibrio splendidus; Vibrio mimicus; Vibrio cincinnatiensis; Vibrio metschnikovii; Vibrio gazogenes; Vibrio mediterranei; Vibrio diazotrophicus; Vibrio fluvialis; Vibrio furnissii; Vibrio proteolyticus; Vibrio nereis; Vibrio tubiashii; Vibrio nigripulchritudo; Vibrio aerogenes; Vibrio orientalis; V. ordalii; Vibrio aestuarianus; and Vibrio carchariae), seven other marine bacteria (Alivibrio; Salinivibrio; Enterovibrio; Grimontia; Photobacterium; Plesiomonas; and Shewanella), and six common bacteria (E. coli; Aeromonas; Salmonella; Pseudomonas; Acinetobacter; and Yersinia). The seven marine bacteria were selected because they were found to exhibit a relatively high sequence homology with the candidate proteins in an overall BLAST with non-Vibrio spp. strains.

\section{Antigenicity and Adhesion Index Analysis of the Candidate Proteins}

Antigenicity refers to the ability of an antigen to be recognized by the immune system. The potential antigenicity of the candidates was estimated through VaxiJen (Doytchinova and Flower, 2007) with a cut-off of 0.4. Adhesion-related OMPs are involved in the pathogenicity of the bacteria and have a greater priority for vaccine development. Vaxign (He et al., 2010) predicts the possible vaccine candidates according to various vaccine design criteria (e.g., sub-cellular location, adhesion, and transmembrane helix). The predication of Vaxign programs has corresponding sensitivity and specificity values of 0.494 and 0.853 (Jaiswal et al., 2013). Proteins with a Vaxign index higher than 0.5 were predicted to be adhesion-related proteins and selected as vaccine candidates.

\section{Surface Proteome of V. parahaemolyticus Analyzed by Trypsin Shaving and Mass Spectrometry}

The surface proteome of $V$. parahaemolyticus was studied by "shaving" the cells with trypsin as described (Rodríguez-Ortega, 2018; Hornburg et al., 2019). Briefly, V. parahaemolyticus (CICC 21617) was cultured in Luria-Bertani broth with $3.0 \% \mathrm{NaCl}$ 


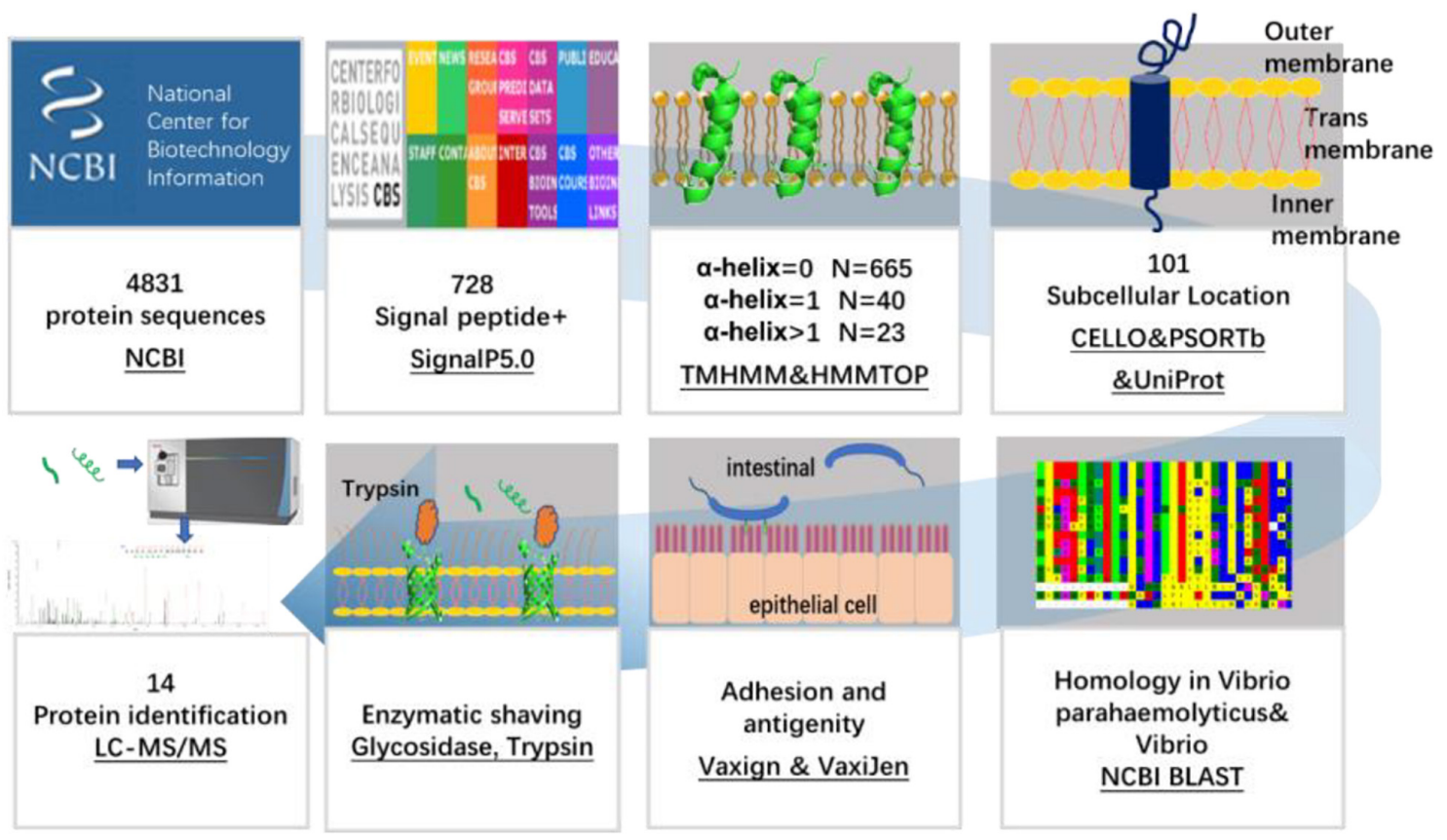

FIGURE 1 | Schematic diagram of a bioinformatical and proteomic approach for the identification of OMPs against V. parahaemolyticus and Vibrio spp.

at $37^{\circ} \mathrm{C}$ until the $\mathrm{OD}_{600}$ was 0.38 , which corresponded to the mid-exponential phase. The culture $(50 \mathrm{~mL})$ was centrifuged at $3,500 \mathrm{~g}, 10 \mathrm{~min}, 4^{\circ} \mathrm{C}$. The pellet was gently suspended in $5 \mathrm{~mL}$ $1 \%$ paraformaldehyde (PFA) (Aladdin Bio-Chem Technology, Shanghai, China) in PBS for $5 \mathrm{~min}$ and washed twice with PBS. One set of the sample was resuspended in $1 \mathrm{~mL}$ shaving buffer $(1 \times$ PBS, $20 \%[\mathrm{w} / \mathrm{v}]$ sucrose, $10 \mathrm{mM} \mathrm{DTT})$ and added with $20 \mu \mathrm{g}$ sequencing grade trypsin (Sigma-Aldrich, St. Louis, MO, United States) in $0.5 \mathrm{~mL} 25 \mathrm{mM}$ ammonium bicarbonate buffer. The treated samples were incubated at $37^{\circ} \mathrm{C}$ in water bath for 15 and $30 \mathrm{~min}$. The control was added with the $0.5 \mathrm{~mL} 25 \mathrm{mM}$ ammonium bicarbonate buffer and incubated at $37^{\circ} \mathrm{C}$ in water bath for $30 \mathrm{~min}$. In a further study, the cells were first treated with endoglycosidase $\alpha$-amylase (Macklin Biochemical Co., Ltd., Shanghai, China), cellulase (solarbio, Beijing, China), pullulanase (Shijiazhuang Ningnuo Trading Co., Ltd., Hebei, China), dextranase (G-CLONE, Biotech Co., Ltd., Beijing, China), and exoglycosidase starch glucosidase (Macklin Biochemical Co., Ltd., Shanghai, China) to reduce the capsule and lipopolysaccharide on the cell surface before "shaving" with trypsin. The cell pellets of $50 \mathrm{~mL}$ culture were resuspended in $5 \mathrm{~mL}$ phosphate buffer ( $\mathrm{pH}$ 5.5) with final concentration of 100 $\mathrm{U} / \mathrm{mL}$ of $\alpha$-amylase $(\alpha-1,4$-linkage), cellulase $(\beta-1,4)$, pullulanase $(\alpha-1,6)$, dextranase $(\beta-1,3 ; \beta-1,4)$, and starch glucosidase $(\alpha-$ $1,4 ; \alpha-1,6$; and $\alpha-1,3)$. The samples were incubated at $50^{\circ} \mathrm{C}$ in water bath for $6 \mathrm{~h}$. The content of saccharides in the supernatant was measured by phenol sulfuric acid method (Cuesta et al., 2003). Then the cells were centrifuged, washed by PBS, and treated with the trypsin by the same way as described above. After centrifugation at $8,000 \mathrm{~g}, 10 \mathrm{~min}, 4^{\circ} \mathrm{C}$, the samples were filtered with a $0.22-\mu \mathrm{m}$ pore-size filter and sent to Sangon Biotech
(Shanghai) Co., Ltd. for the $30 \mathrm{~min}$ LC-MS/MS analysis. The MS/MS data was acquired when the precursor ion signal was greater than $120^{\circ}$ and the charge number was $+2 \sim+5$. The database used for searching was the proteome reference database of $V$. parahaemolyticus O3:K6 (strain RIMD 2210633) in UniProt. Two trial of the experiments were conducted and three replicates of each sample were tested.

\section{Structure Simulation of the Candidate Proteins}

The three-dimensional protein structure was simulated by the online servers, I-TASSER (Yang et al., 2015), and TrRosetta (Yang et al., 2020). I-TASSER is an iterative template-based fragment assembly simulation server. TrRosetta builds the protein structure based on direct energy minimization with a restrained Rosetta. Briefly, the sequence of the candidate protein was inputted and submitted to the server with an automatic model, and a high-quality simulated protein structure is obtained when the C-score is more than -1.5 in I-TASSER, the C-score is greater than -1.5 , and a Tm score more than 0.5 in TrRosetta (Xu and Zhang, 2010).

\section{RESULTS}

\section{Bioinformatical Identification of V. parahaemolyticus Outer Membrane Proteins}

In this study, screening of the 4,831 proteins encoded by $V$. parahaemolyticus RIMD 2210633 revealed 728 proteins that 

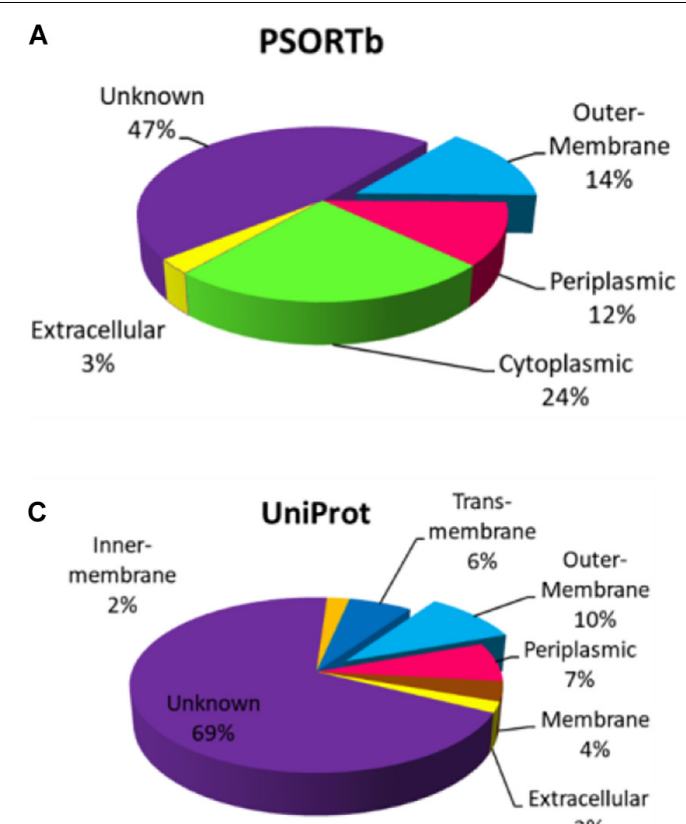
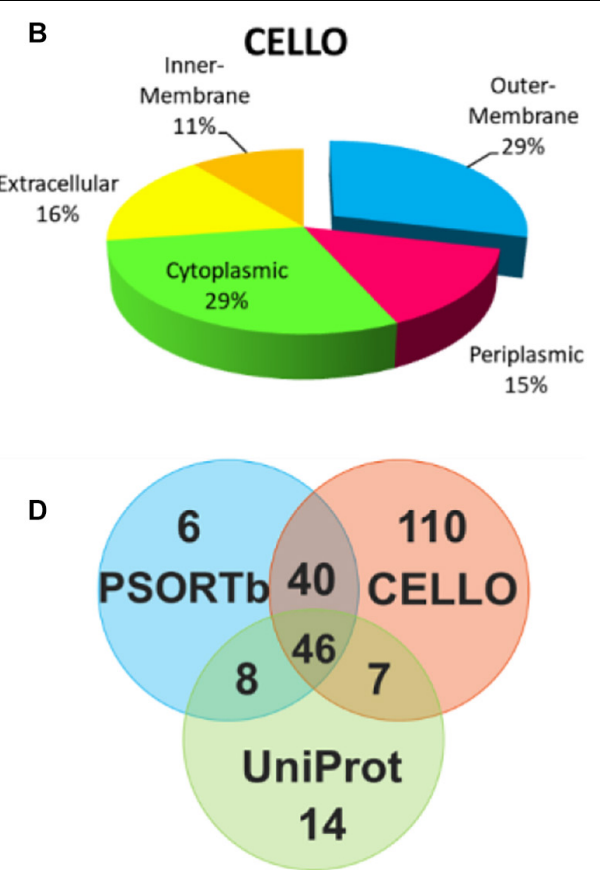

FIGURE 2 | Subcellular location analysis of the 705 proteins by PSORTb (A), CELLO (B), and UniProt (C). (D) Mapping the OMPs predicted by three methods.

had a signal peptide sequence in the $\mathrm{N}$-terminal region. Among them, 505 were general secretory pathway (Sec) signal peptides, 21 were twin-arginine translocation (TAT) signal peptides, and 202 were lipoprotein signal peptides. The outer membrane proteome of bacteria is dominated by a $\beta$-barrel with no TM $\alpha$-helix or $\alpha$-helical membrane proteins with one or more TM $\alpha$-helix (Rollauer et al., 2015). TMHMM 2.0 and HMMTOP showed 665 proteins had no TM $\alpha$-helix and 40 proteins had one TM $\alpha$-helix, which indicated they were primarily $\beta$ barrel proteins. The $\beta$-barrel proteins are mainly porin proteins that occur only in the outer membranes of bacteria and are involved in metabolite and protein transport, osmotic pressure regulation, and numerous signaling processes (Wimley, 2003). The other 23 proteins were excluded for further analysis due to a substantial challenge of multi-pass TM protein expression in a prokaryotic expression system as reported by Serruto (Serruto et al., 2004).

To select OMPs that are primarily involved in the host environment interaction and exclude the cytoplasmic proteins (Sanober et al., 2017), the subcellular localization of the proteins was predicted using three parallel online approaches. Of the $705 \mathrm{~V}$. parahaemolyticus proteins, PSORTb, CELLO, and UniProt revealed that 100 (14.2\%), 203 (28.8\%), and 75 $(10.6 \%)$, respectively, were located in the outer membrane region (Figure 2). As shown in Figure $\mathbf{2 A}$ and $\mathbf{C}, 47$ and $69 \%$ of the proteins were not predicted or annotated with clear subcellular location by PSORTb and UniProt. It's noteworthy that UniProt annotated 26 membrane proteins and 45 TM proteins, some of which may also be OMPs. To balance the prediction coverage and confidence of prediction, the 101 proteins (Supplementary Table 1) that were predicted as OMPs by at least two servers were selected for further analysis.

\section{Homology and Adhesion of V. parahaemolyticus and Vibrio spp. Proteins}

The sequence homology of 101 OMPs identified in $V$. parahaemolyticus RIMD 2210633 with heterogenous serotypes and isolates of $V$. parahaemolyticus was analyzed by BLAST in NCBI. The results showed that these OMPs are conserved $(>98 \%)$ in $V$. parahaemolyticus. The BLAST of the identified OMPs with 32 other Vibrio species, seven closely related marine bacteria, and six other common bacteria showed that $70 \%$ of the OMPs (e.g., previously reported OmpA, OmpW, and OmpU) had relatively high but uneven sequence homology with other Vibrio species and marine bacteria, such as Photobacterium, Shewanella, Aliivibrio, Salinivibrio, Enterovibrio, and Grimontia. Interestingly, most of the OMPs were found to share a high sequence homology with nine Vibrio species, including $V$. alginolyticus, $V$. diabolicus, $V$. antiquaries, $V$. campbelli, V. harveyi, V. owensii, V. rotiferianus, $V$. jasicida, and $V$. natriegens, which indicated a close genetic relationship between these strains. Notably, $V$. diabolicus and $V$. antiquaries are isolated from a deep-sea hydrothermal vent environment, and their prevalence and pathogenicity in coastal systems and seafood samples remains unclear (Turner et al., 2018).

However, we did identify 19 OMPs that exhibited relatively good specificity for $V$. parahaemolyticus. As listed in Table 1, six OMPs, including OmpA-like domain-containing protein (VPA0731), outer membrane protein (VPA0316), AraC/XylS 
TABLE 1 | The homology of differential OMPs of $V$. parahaemolyticus identified by bioinformatic tools.

\begin{tabular}{|c|c|c|c|c|c|c|}
\hline \multirow[t]{2}{*}{ Accession } & \multirow[t]{2}{*}{ Description } & \multirow[t]{2}{*}{$\alpha$-Helix } & \multirow[t]{2}{*}{ Adhesion } & \multicolumn{3}{|c|}{ Sequence homology } \\
\hline & & & & V. parahaemolyticus & 32 Species of Vibrio spp. & non-Vibrio strains \\
\hline \multirow[t]{2}{*}{ NP_800241.1 } & OmpA-like domain-containing protein & 0 & 0.200 & $92 \% 100$ & 74\%, V. harveyi & $N$ \\
\hline & & & & & $74 \%$, V. diabolicus & \\
\hline NP_798570.1 & hypothetical protein & 1 & 0.125 & $91 \% 280$ & $<66 \%$, nine species ${ }^{a}$ & $N$ \\
\hline NP_800966.1 & hypothetical protein & 1 & 0.103 & $97 \% 430$ & $<75 \%$, nine species & $N$ \\
\hline NP_799826.1 & Outer membrane protein & 0 & 0.686 & $92 \% 70$ & $<76 \%$, nine species & $N$ \\
\hline NP_797233.1 & AraC/XylS family transcription factor & 1 & 0.250 & $99 \% 500$ & $<81 \%$, nine species & $N$ \\
\hline NP_800058.1 & hypothetical protein & 0 & 0.353 & $98 \% 73$ & $<85 \%$, nine species & $N$ \\
\hline NP_799595.1 & hypothetical protein & 0 & 0.325 & $99 \% 54$ & 99\%, V. diabolicus & Photobacterium 50\% \\
\hline \multirow[t]{2}{*}{ NP_800886.1 } & hypothetical protein & 0 & 0.586 & $97 \% 20$ & $83 \%$, V. cholerae & Shewanella et al. $57 \%$ \\
\hline & & & & & $97 \%$, V. mimicus & \\
\hline \multirow[t]{2}{*}{ NP_799735.1 } & Putative efflux pump channel protein & 0 & 0.565 & $97 \% 344$ & $97 \%, V$. alginolyticus & Shewanella et al. 55\% \\
\hline & & & & & $80 \%$, V. natriegens & \\
\hline NP_797735.1 & hypothetical protein & 0 & 0.468 & $95 \% 287$ & $87 \%, V$. natriegens & Photobacterium $74 \%$ \\
\hline NP_801014.1 & CsuD protein & 0 & 0.411 & $97 \% 444$ & $87 \%, V$. alginolyticus, $V$. diabolicus, $V$. antiquaries & Enterovibrio $60 \%$ \\
\hline NP_797599.1 & MtrC & 0 & 0.430 & $97 \% 467$ & $96 \%, V$. diabolicus, V. antiquaries & Enterovibrio 52\% \\
\hline OAR40234.1 & flagellin & 0 & 0.393 & $98 \% 304$ & $<81 \%$, nine species & Grimontia et al. $47 \%$ \\
\hline NP_797143.1 & OmpA & 0 & 0.311 & $97 \% 78$ & $<88 \%$, nine species & Photobacterium $50 \%$ \\
\hline NP_799317.1 & vitamin B12 receptor & 0 & 0.608 & $97 \% 154$ & $<89 \%$, nine species & Aeromonas et al. $41 \%$ \\
\hline NP_799752.1 & OMP_b-brl domain-containing protein & 0 & 0.611 & $95 \% 122$ & $<89 \%$, nine species & Grimontia 67\% \\
\hline NP_800976.1 & TonB system receptor & 0 & 0.508 & $97 \% 405$ & $<89 \%$, nine species & Alivibrio et al. $59 \%$ \\
\hline
\end{tabular}

${ }^{a}$ homology was only found with the nine species including $V$. alginolyticus, V. diabolicus, V. antiquaries, V. campbelli, V. harveyi, V. owensii, V. rotiferianus, V. jasicida, and V. natriegens. 
family transcription factor (VP0854), and three hypothetical proteins (VP2191, VPA1456, and VPA0548), shared negligible homology $(<32 \%)$ with non-Vibrio spp. and relatively low homology $(<85 \%)$ with two to nine Vibrio species. Eleven OMPs shared a low homology $(38-74 \%)$ with several non-Vibrio strains, six of which (NP_799595.1, NP_800886.1, NP_799735.1, and NP_797735.1, CsuD protein, and MtrC) only had a high homology $(83$ - 99\%) with one to three Vibrio species ( $V$. alginolyticus, $V$. diabolicus, and V.antiquaries). The last five OMPs (flagellin, OmpA, NP_799317.1, NP_799752.1, and NP_800976.1) shared a sequence homology of less than $89 \%$ with the closely related nine Vibrio species. There are two differential OMPs that are not listed in Table 1. The long-chain fatty acid transport protein (NP_798592.1) was only found to display a high homology (93\%) with $V$. alginolyticus, $V$. diabolicus, $V$. antiquaries, and $V$. rotiferianus and shares a $66 \%$ identify with Photobacterium and Shewanella. The outer membrane porin protein (NP_797387.1) is specific to the $V$. parahaemolyticus, $V$. diabolicus, $V$. antiquaries, $V$. campbelli, $V$. harveyi, and $V$. jasicida and was not found in the non-Vibrio spp. strains.

Table 2 shows that there were 15 OMPs that shared at least a $65 \%$ sequence homology across 23 or more species out of the 32 Vibrio species. These proteins are widely conserved in Vibrio spp. because they are involved in the fundamental structure or biological function, such as the flagellar motor protein complex (e.g., flgH1 and motY), new OMPs assembly (e.g., BamA), polysaccharide assembly (e.g., LptD), fimbriae assembly (NP_799125.1 and NP_796512.1), the TolC subfamily within the efflux systems (NP_798013.1, NP_798092.1, and NP_796804.1), maintaining the outer membrane integrity (VacJ), polysaccharide binding, and carbohydrate metabolic process (NP_797134.1). While the protective efficacy of proteins OmpW (Mao et al., 2007), OmpK (Li et al., 2010b), LptD (Zha et al., 2016), and Pal (Li et al., 2010a) has already been studied. The other OMPs that are conserved in more Vibrio species were newly identified in this study.

Figure $\mathbf{3 A}$ shows the relationship of the identified OMPs with the number of $\alpha$-helix structure, $V$. parahaemolyticus differential OMPs, Vibrio spp. conserved OMPs. Most candidate OMPs had no TM $\alpha$-helix, which indicated a dominant $\beta$-barrel structure. The analysis of protein antigenicity by VaxiJen showed that 96 out of the 101 proteins exhibited a higher score than the cut-off $(>0.4)$, which indicated the identified OMPs were potentially antigenic proteins. Adhesion proteins help bacteria attach to the host cell receptors and promote colonization. Creating a vaccine and generating associated neutralizing antibodies against adhesion proteins is effective in preventing bacterial infections at an early stage (Wizemann et al., 1999). The results of the analysis by Vaxign showed that 43 OMPs had adhesion probability higher than 0.5 and were predicated to be related to adhesion (Figure 3B). Among these, eight OMPs (outer membrane protein NP_799826.1, hypothetical protein NP_800886.1, putative efflux pump channel protein NP_799735.1, vitamin B12 receptor NP_799317.1, OMP_b-brl domain-containing protein NP_799752.1, and TonB system receptor NP_800976.1) were more specific to $V$. parahaemolyticus (Table 1) and four OMPs (flgH,
LptD, OmpK, and OmpW) were widely conserved in Vibrio spp. (Table 2).

\section{Surface Proteins Identified by Enzymic Shaving and Mass Spectrometry}

Trypsin was specific to hydrolyze carboxyl side of lysine and arginine residues in polypeptide chain and was widely used in proteomic studies (Olaya-Abril et al., 2014). To experimentally study the OMPs on the cell surface, trypsin was first used to enzymatically shave off the intact cells. The results of LC-MS/MS showed although little intracellular proteins were observed for the control sample without trypsin, there were no known OMPs identified in the experimental group after 30 min shaving except some intracellular proteins (Table 3). The identified BamD that annotated as OMP actually faced the periplasm side of the outer membrane (Han et al., 2016). Vibrio was known to express the capsule ( $\mathrm{K}$ antigen) on the cell to adapt the environment and escape the phagocytosis (Pettis and Mukerji, 2020). This may limit the availability of OMPs to trypsin digestion. Many studies have shown that the capsule or lipopolysaccharides consist of $\alpha-1,3, \alpha-1,2, \alpha-1,4, \beta-1,4, \beta-1,3, \alpha-1,5, \beta-1,5, \alpha-1,6$, and $\beta$ 1,6 glycosidic bond (Chen et al., 2007). The endoglycosidases were known to cleavage the specific glycosidic bond inside the polysaccharide and have been reported to reduce the capsule level of Streptococcus pneumoniae (Middleton et al., 2018). With the combination of four endoglycosidase and one exoglycosidase in a one-step enzymatical digestion of the intact cells, the sugar content in the supernatant increased from 4.89 to $12.90 \mathrm{mg} / 100 \mathrm{~g}$ after $6 \mathrm{~h}$ (Supplementary Figure 1). This indicated the reduction of exopolysaccharides on the cell. The following enzymatical shaving of the intact cells by trypsin showed more intracellular proteins in both the positive and control group were observed, but peptides of some proteins were mainly observed in the experimental group and further analysis with UniProt confirmed there were 14 OMPs identified. These OMPs were OmpA (VPA1186, VPA0248, and VP0764), Omp (VPA0166), OmpU (VP2467), BamA (VP2310), TolC (VP0425), GspD (VP0133), OmpK (VP2362), lpp (VPA1469), Pal (VP1061), 5'-nucleotidase (VP0748), agglutination protein (VP1634), and putative iron (III) compound receptor (VPA1435).

\section{Protein Structure of the Omps Identified by Both Reverse Vaccinology and Surface Proteome}

The results in Tables 2, 3 revealed that OmpK, agglutination protein, Pal, TolC, GspD, and BamA were identified by the bioinformatical screening of the encoded protein database and enzymatical shaving the intact cells of $V$. parahaemolyticus. Protein simulation by I-TASSER and TrRosetta (Figure 4) showed that all these protein structures had an estimated TM score $\geq 0.5$ and C-score more than -1.5 . The tolC super family protein agglutination protein (VP1634) and Tolc (VP0425) had a typical $\beta$-barrel domain on the outer membrane and $\alpha$-helical colied coli structure protrude deep into the periplasm (Zgurskaya et al., 2011). The GspD belong to the type II secretory system also had a similar structure which promote the secretion of 
TABLE 2 | The homology of differential OMPs of Vibrio spp. identified by bioinformatic tools.

\begin{tabular}{|c|c|c|c|c|c|c|}
\hline \multirow[t]{2}{*}{ Accession } & \multirow[t]{2}{*}{ Description } & \multirow[t]{2}{*}{$\alpha$-Helix } & \multirow[t]{2}{*}{ Adhesion } & \multicolumn{3}{|c|}{ Sequence homology } \\
\hline & & & & V. parahaemolyticus & 32 Species of Vibrio spp. & non-Vibrio strains $^{\mathrm{a}}$ \\
\hline NP_798689.1 & Outer membrane protein assembly factor YaeT BamA & 0 & 0.439 & $98 \% 63$ & $>70 \%, 31$ species & $53-78 \%$ \\
\hline NP_796804.1 & Outer membrane channel protein Tolc & 0 & 0.427 & $91 \% 62$ & $>70 \%, 31$ species & $51-69 \%$ \\
\hline NP_799125.1 & Fimbrial assembly protein & 0 & 0.222 & $93 \% 106$ & $>65 \%, 31$ species & $52-70 \%$ \\
\hline NP_796512.1 & General secretion pathway protein D & 0 & 0.142 & $93 \% 49$ & $>75 \%, 31$ species & $54-70 \%$ \\
\hline NP_797161.1 & Flagellar basal body L-ring protein & 0 & 0.681 & $99 \% 33$ & $>70 \%, 30$ species & Salinivibrio 58\% \\
\hline NP_798092.1 & Hypothetical protein & 0 & 0.336 & $93 \% 160$ & $>65 \%, 29$ species & $52-62 \%$ \\
\hline NP_798593.1 & VacJ lipoprotein & 0 & 0.289 & $99 \% 99$ & $>65 \%, 29$ species & $50-62 \%$ \\
\hline NP_796718.1 & LPS-assembly protein LptD & 0 & 0.649 & $92 \% 117$ & $>60 \%, 29$ species & $44-48 \%$ \\
\hline NP_798013.1 & Agglutination protein tolC super family & 0 & 0.411 & $99 \% 94$ & $>70 \%, 28$ species & $51-68 \%$ \\
\hline NP_798490.1 & Sodium-type flagellar protein MotY & 0 & 0.255 & $94 \% 60$ & $>70 \%, 28$ species & $62-65 \%$ \\
\hline SUQ25448.1 & Outer membrane protein OmpK & 0 & 0.763 & $79 \% 43$ & $>65 \%, 27$ species & $65-80 \%$ \\
\hline NP_797646.1 & Lipoprotein & 1 & 0.416 & $97 \% 26$ & $>70 \%, 25$ species & $40-70 \%$ \\
\hline NP_797440.1 & Peptidoglycan-associated lipoprotein Pal & 0 & 0.333 & $99 \% 24$ & $>70 \%, 25$ species & $61-64 \%$ \\
\hline NP_797134.1 & Chitobiase & 0 & 0.386 & $99 \% 380$ & $>70 \%, 24$ species & $57-74 \%$ \\
\hline NP_799606.1 & Outer membrane protein OmpW & 0 & 0.774 & $90 \% 51$ & $>65 \%, 23$ species & Grimontia 71\% \\
\hline
\end{tabular}

These non-Vibrio strains include Alivibrio Sp., Salinivibrio Sp., Enterovibrio Sp., Grimontia Sp., and Photobacterium Sp. 

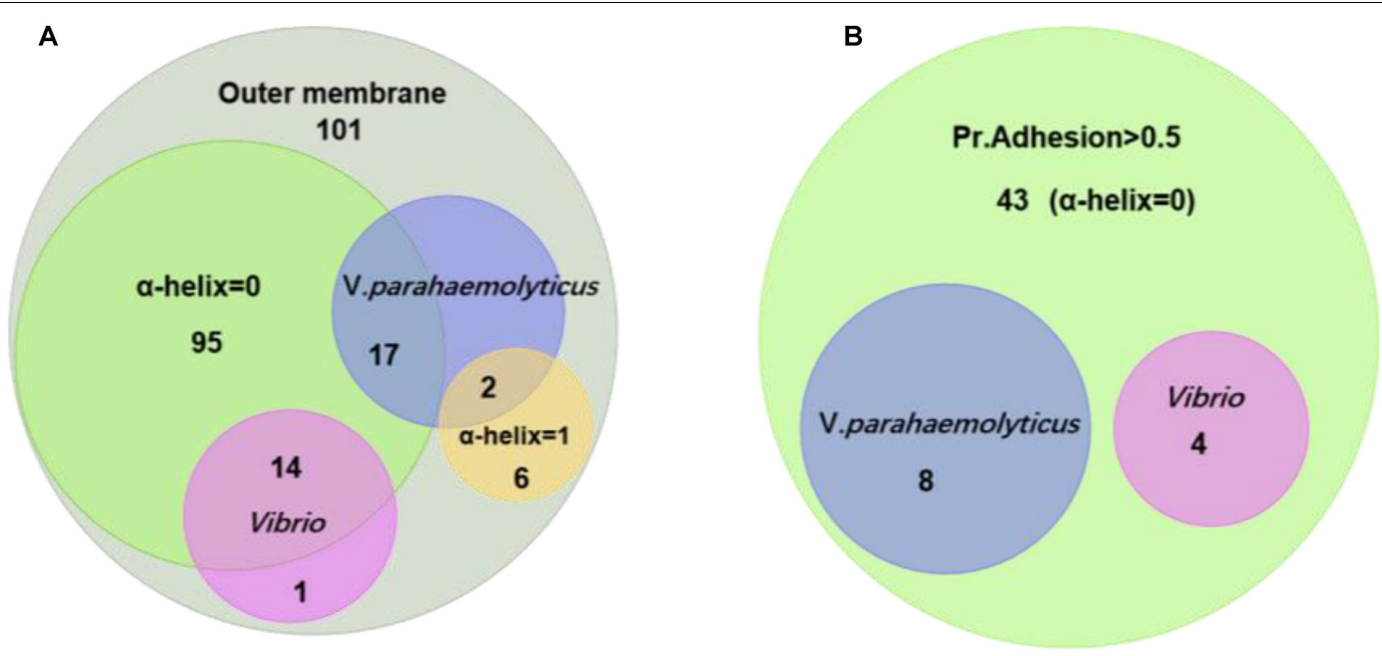

FIGURE 3 | Homology and adhesion. (A) The distribution and crossover of 101 outer membrane protein candidates. There are 15 proteins that are homologous in Vibrio; 19 proteins that are homologous in V. parahaemolyticus; 95 proteins that have no TM $\alpha$-helix; and six proteins that have one TM $\alpha$-helix. (B) There are 43 proteins that have a Pr. adhesion score of more than 0.5; four proteins are homologous in Vibrio; and eight proteins are homologous in $V$. parahaemolyticus.

protein. The BamA and OmpK structure had a common $\beta$ barrel structure of a porin protein, which are involved in protein assembly and receptor.

\section{DISCUSSION}

The novel OMPs as immunogens for preparing diagnostic antibodies and subunit vaccine against $V$. parahaemolyticus and Vibrio spp. are of interest in aquaculture and food safety. Traditional study of the major OMPs was unsystematic and time consuming. Based on bioinformatic tools with different filter thresholds and applications, reverse vaccinology processes a large quantity of genetic or proteomic data and minimize the targets in the following in vivo experiments. The surface proteome based on enzymatical shaving of the extracellular peptides of the cells and massive identification of the peptides provides an experimental insight of the surface available OMPs. The two comprehensive and complementary strategies were both adopted in this work to study the diagnostic surface antigens of $V$. parahaemolyticus and Vibrio species.

For the bioinformatic analysis, signal sequences presented at the N-terminal were first predicted because most bacterial OMPs have an N-terminal signal sequence that promotes protein secretion (Gao et al., 2016). Our result identified three different signal peptides. The Sec signal peptide directs unfolded protein translocation across the plasma membrane in prokaryotes (Tsirigotaki et al., 2017). TAT signal peptide actively translocate the folded proteins across the lipid bilayer of the membrane (Armenteros et al., 2019) and are generally longer and less hydrophobic (Berks, 2015). The lipoprotein signal peptides play an important role in the maturation of bacterial lipoprotein (Kitamura and Wolan, 2018), many of which are surface-exposed (Wilson and Bernstein, 2016). To cross-check the subcellular location of candidate proteins, three parallel prediction tools were used in this study. Among them, PSORTb was reported to be the most precise bacterial localization prediction tool available based on a report in 2010 (Yu et al., 2010). The results in Figure 2D showed that when CELLO and PSORTb, CELLO and UniProt, PSORTb and UniProt were used to cross-check, 86, 53, and 54 proteins were, respectively, predicted as OMPs. Parallel use of the three prediction tools and retaining the proteins predicted by two or three methods obtained more candidate OMPs (101) with better coverage and accuracy.

The homology analysis of the $V$. parahaemolyticus OMP candidates by BLASTp revealed a relatively conserved and complex genetic relationship with the other Vibrio species and some marine bacteria, which provide the insight of possible cross-reaction or cross-protection with these OMPs. Based on the overall homology analysis, 15 OMPs that are broadly conserved in the genus of Vibrio were identified. Four of them were predicted to be adhesion proteins and were potentially multivalent subunit vaccine candidates against vibriosis. Many of these conserved candidate proteins are involved in some significant function. For example, BamA participates in the delivery of extracellular membrane proteins and is the core component of the $\beta$-barrel assembly machinery BAM complex (Singh et al., 2017). Peptidoglycan-associated lipoprotein (Pal) stabilizes the outer membrane by providing a non-covalent biding through the peptidoglycan layer (Parsons et al., 2006). The general secretion pathway protein $(\mathrm{GspD})$ in $V$. cholerae directly involves the production of rugose polysaccharide and the secretion of cholera toxin and hemolysin (Ali et al., 2000). The TolC family protein provides a channel for the cell to connect with the external environment during export and plays a role in the bacterial efflux pumps (Koronakis et al., 2000). The protein $\mathrm{LptD}$ is responsible for lipopolysaccharide transport and insertion into the outer membrane (Dong et al., 2014). The flagellar basal body L-ring protein (flgH), sodium-type flagellar protein motY, and fimbrial assembly protein involved in motility 
TABLE 3 | The identified OMPs in V. parahaemolyticus O3:K6 (RIMD 2210633) by proteomic analysis.

\begin{tabular}{|c|c|c|c|c|c|c|c|c|}
\hline Accession & Description & Function & Mw (kD) & Peptide $\left(-^{a}\right)$ & Peptide $\left(\mathrm{T}^{\mathrm{b}}\right)$ & Peptide $\left(G^{c}\right)$ & Peptide $\left(G+T^{d}\right)$ & Bioinformatics \\
\hline NP_797127.1 & 5'-nucleotidase & Degradation for nutrition & 62.175 & - & - & 1 & 1 & + \\
\hline NP_798741.1 & OmpK & Receptor for vibriophage & 29.877 & - & - & - & 2 & + \\
\hline NP_800979.1 & Outer membrane lipoprotein (lpp) & Distance controls of membranes & 8.671 & - & - & - & 1 & + \\
\hline NP_800945.1 & Putative iron (III) compound receptor & Siderophore uptake transporter & 77.059 & - & - & - & 1 & + \\
\hline NP_800696.1 & OmpA & Porin activity & 36.014 & - & - & - & 5 & + \\
\hline NP_799758.1 & OmpA & Porin activity & 35.553 & - & - & - & 9 & + \\
\hline NP_799676.1 & Putative Omp & Porin activity & 37.974 & - & - & 1 & 4 & + \\
\hline NP_798846.1 & OmpU & Passive diffusion of small molecules & 36.285 & - & - & - & 10 & + \\
\hline NP_798689.1 & BamA & Omp assembly complex & 90.053 & - & - & - & 3 & + \\
\hline NP_798013.1 & Agglutination protein & Efflux transmembrane transporter & 48.69 & - & - & - & 1 & + \\
\hline NP_797440.1 & $\mathrm{Pal}$ & Division and cell integrity & 18.713 & - & - & 1 & 3 & + \\
\hline NP_797143.1 & OmpA & Porin activity & 34.073 & - & - & 2 & 9 & + \\
\hline NP_796804.1 & TolC & Efflux transmembrane transporter & 47.983 & - & - & - & 8 & + \\
\hline NP_796512.1 & GspD & Protein secretion by the T2S & 73.317 & - & - & - & 1 & + \\
\hline
\end{tabular}

${ }^{a}$ Control group treated with only the shaving buffer of trypsin (1 $\times$ PBS, 20\% [W/V] sucrose, $10 \mathrm{mM}$ DTT).

${ }^{b}$ Experimental group treated with trypsin in shaving buffer.

${ }^{c}$ Experimental group treated with glycosidases in phosphate buffer ( $\mathrm{pH}$ 5.5) and the shaving buffer of trypsin.

${ }^{d}$ Experimental group treated with glycosidases in phosphate buffer (pH 5.5) and trypsin in shaving buffer. 


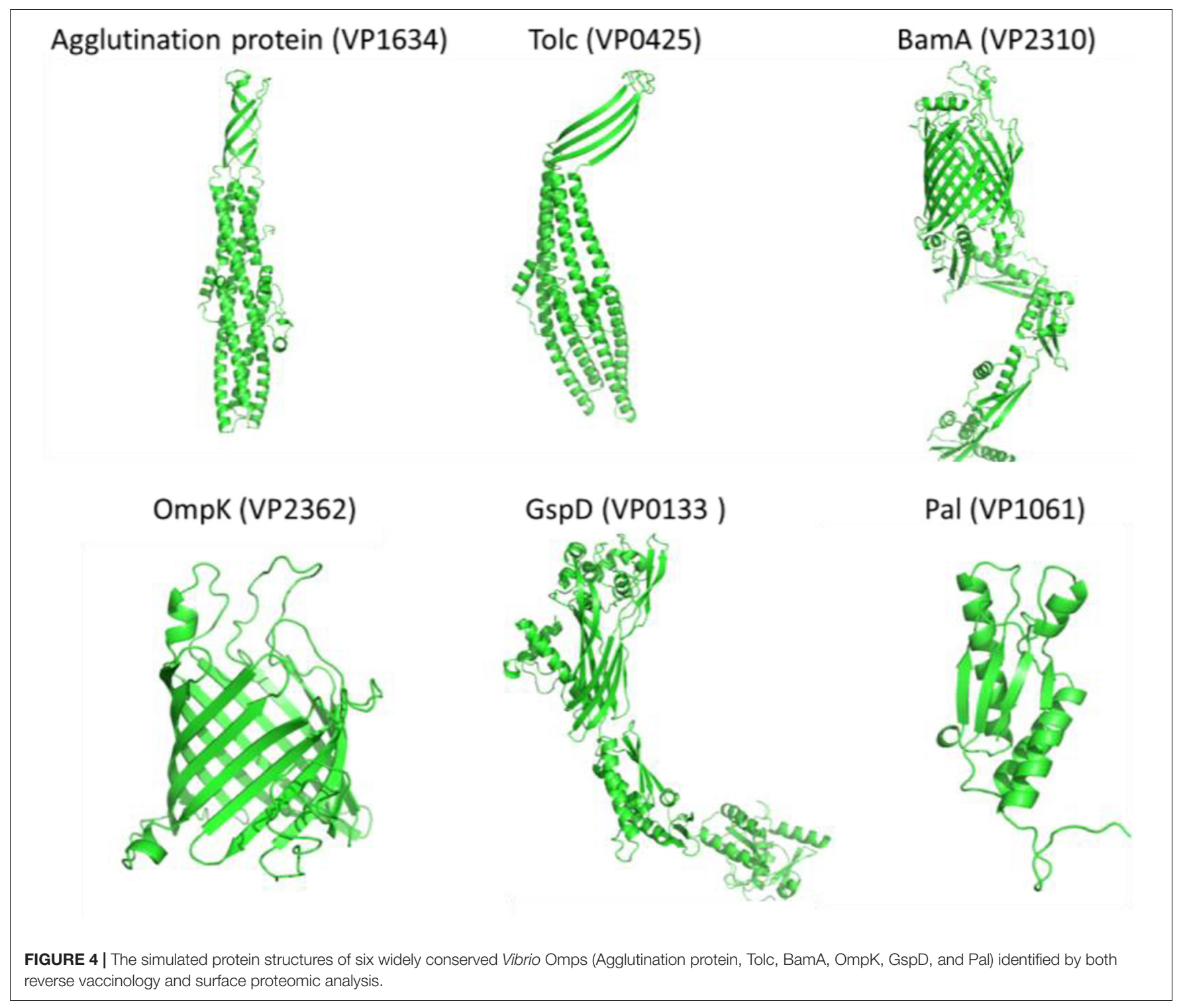

and adhesion (Rehman et al., 2019). The OmpK and OmpW are porin proteins that are involved in the transport of small hydrophobic molecules across the bacterial outer membrane (Hong et al., 2006).

Due to the complex homology, completely specific $V$. parahaemolyticus OMPs were not found, but 19 differential OMPs were obtained in this study. The CsuD protein and MtrC (Beliaev et al., 2001), respectively, has fimbrial usher porin activity and terminal $\mathrm{Fe}$ (III) reductase activity. Vitamin B12 receptor and TonB system receptor play a role in nutrition uptake and transportation (Köster, 2001). OmpA and OmpA like protein function as an adhesin and invasin, participate in biofilm formation, and serve as a bacteriophage receptor (Smith et al., 2007). The AraC-XylS family proteins are known as transcriptional proteins regulator (Tobes and Ramos, 2002). Further analysis of the structure, topology, and sequence homology of these OMPs may reveal the specific extracellular epitopes for $V$. parahaemolyticus and overcome the challenge of discovering diagnostic targets.

Previous immunoproteomic work performed by Li et al. (2010a) identified the putative iron (III) compound receptor (VPA1435), TolC (VP0425), and OmpA (VPA0764), conserved hypothetical protein (VP2850), BamA (VP2310), and Pal (VP1061) as the immunogenic OMPs of $V$. parahaemolyticus. Similar work described by another group identified the lipopolysaccharide-assembly protein, LptD, hypothetical protein (VP0802), Maltoporin (LamB), OmpA, OmpU, and OmpK, as well as hypothetical VP1243 and VP0966 (Li et al., 2014). Recently, Yonekita et al. (2020) reported that a diagnostic antibody prepared against a whole cell antigen was specific to 12 serotypes of $V$. parahaemolyticus and did not react with the other six Vibrio species. The antigen was identified to be outer membrane lipoprotein (NP_800979.1 and VPA1469). All of these proteins were predicted as OMPs in this study, except VP2850, 
VP1243, and VP0966, which were only predicted by CELLO. Importantly, we confirmed the homology of these OMPs among the 32 species of Vibrio and closely related non-Vibrio strains at the sequence level. LptD, Pal, BamA, TolC, OmpK, and OmpW (Table 2) were conserved in at least 23 Vibrio species. The lipo protein VPA1469 was also found to have a high homology with $V$. campbelli, $V$. harveyi $V$. owensii, and V. rotiferianus. Apart from these Omps, we identified new OMPs that were conserved in Vibrio spp. or relatively specific to $V$. parahaemolyticus for development of better diagnostic tools and vaccines.

In our surface proteome study, direct shaving of PFA fixed $V$. parahaemolyticus cells resulted in no OMPs but some cytoplasmic proteins, which were also found in many previous surface proteome studies of Gram-negative strains (Fagerquist and Zaragoza, 2018). This is mainly because the soft cell membrane is more vulnerable to osmotic lysis (Olaya-Abril et al., 2014). Combination of four endoglycosidases and an exoglycosidase in a one-step digestion reduced capsule level of $V$. parahaemolyticus in this study. The following shaving with trypsin and analysis indicated 14 OMPs, also identified in the reverse vaccinology strategy, may have a better accessibility on the cell surface. Rodríguez-Ortega found the number of surface-exposed proteins for group A Streptococcus varied from strain to strain and it's mostly because of the different capsule content (Rodríguez-Ortega et al., 2006). Our results also suggested a steric hindrance effect of the capsular polysaccharide, which was widely expressed by Vibrio species, most likely masked the OMPs from enzymatical shaving of the intact cell. The mask of many OMPs by capsule may also limit its reaction with antibodies, which should be considered in immunodetection and vaccine development. The expression of capsule of Vibrio was regulated by temperature, divalent cation $\left(\mathrm{Ca}^{2+}\right.$ and $\left.\mathrm{Mn}^{2+}\right)$, oxygen content, and host environment (Pettis and Mukerji, 2020). Further study of the surface proteome of $V$. parahaemolyticus with minimal capsule expression will elucidate more stably expressed OMPs and the optimal enrich broth for the immunodetection of $V$. parahaemolyticus from various environmental samples.

In short, reverse vaccinology analysis of the surface proteins based on the protein database of $V$. parahaemolyticus in NCBI comprehensively predicted 101 OMPs and revealed 19 differential OMPs of $V$. parahaemolyticus and 15 conserved OMPs of Vibrio spp. Surface proteome study of $V$. parahaemolyticus by enzymatical shaving of the exopolysaccharide and proteins on the intact cell identified 14 OMPs, which mainly belong to the conserved OMPs of Vibrio spp. Future study of the capsule expression level, surface

\section{REFERENCES}

Ali, A., Johnson, J. A., Franco, A. A., Metzger, D. J., Connell, T. D., Morris, J. G., et al. (2000). Mutations in the extracellular protein secretion pathway genes (eps) interfere with rugose polysaccharide production in and motility of Vibrio cholerae. Infect. Immun. 68, 1967-1974. doi: 10.1128/iai.68.4.1967-1974. 2000

Aly, S. M., Eissa, A. E., Elbanna, N. I., and Albutti, A. (2020). Efficiency of monovalent and polyvalent Vibrio alginolyticus and Vibrio parahaemolyticus proteome of $V$. parahaemolyticus under various environments, and the interaction of $V$. parahaemolyticus with antibodies against these Omps will further reveal the diagnostic surface antigen or epitopes of $V$. parahaemolyticus and Vibrio spp.

\section{DATA AVAILABILITY STATEMENT}

The datasets presented in this study can be found in online repositories. The names of the repository/repositories and accession number(s) can be found in the article/ Supplementary Material.

\section{ETHICS STATEMENT}

This article does not contain any studies with human participants or animal experiments.

\section{AUTHOR CONTRIBUTIONS}

WW and SP initiated the study. JL and WW mainly conducted the experiment, analyzed the data, and wrote the manuscript. JL, LL, SG, and QY participated the data analysis. LG revised the manuscript. All the authors read and approved the manuscript.

\section{FUNDING}

This work is supported by the National Natural Science Foundation of China (Grant No. 31701680), the Natural Science Foundation of Jiangsu Province of China (Grant No. SBK2017041308), the Open Project Program of Key Laboratory for Analytical Science of Food Safety and Biology, Ministry of Education (FS2003), Open-end Funds of Jiangsu Key Laboratory of Marine Biotechnology, Jiangsu Ocean University (HS2019003), Postgraduate Research and Practice Innovation Program of Jiangsu Province(KYCX19_2289), and also funded by the Priority Academic Program Development of Jiangsu Higher Education Institutions.

\section{SUPPLEMENTARY MATERIAL}

The Supplementary Material for this article can be found online at: https://www.frontiersin.org/articles/10.3389/fmicb. 2020.625315/full\#supplementary-material

vaccines on the immune response and protection in gilthead sea bream, Sparus aurata (L.) against vibriosis. Fish Shellfish Immunol. 2020:11. doi: 10.1016/j.fsi. 2020.10.011

Armenteros, J. J. A., Tsirigos, K. D., Sønderby, C. K., Petersen, T. N., Winther, O., Brunak, S., et al. (2019). SignalP 5.0 improves signal peptide predictions using deep neural networks. Nat. Biotechnol. 37, 420-423. doi: 10.1038/s41587-0190036-z

Baker-Austin, C., Oliver, J. D., Alam, M., Ali, A., Waldor, M. K., Qadri, F., et al. (2018). Vibrio spp. infections. Nat. Rev. Dis. Primers 4:8. 
Baliga, P., Shekar, M., and Venugopal, M. N. (2018). Potential outer membrane protein candidates for vaccine development against the pathogen Vibrio anguillarum: A reverse vaccinology based identification. Curr. Microbiol. 75, 368-377. doi: 10.1007/s00284-017-1390-z

Beliaev, A. S., Saffarini, D. A., Mclaughlin, J. L., and Hunnicutt, D. (2001). MtrC, an outer membrane decahaem c cytochrome required for metal reduction in Shewanella putrefaciens MR-1. Mol. Microbiol. 39, 722-730. doi: 10.1046/j. 1365-2958.2001.02257.x

Berks, C. B. (2015). The twin-arginine protein translocation pathway. Annu. Rev. Biochem. 84, 843-864. doi: 10.1146/annurev-biochem-060614-034251

Bonnin-Jusserand, M., Copin, S., Le Bris, C., Brauge, T., Gay, M., Brisabois, A., et al. (2019). Vibrio species involved in seafood-borne outbreaks (Vibrio cholerae, $V$. parahaemolyticus and $V$. vulnificus): Review of microbiological versus recent molecular detection methods in seafood products. Crit. Rev. Food Sci. Nutr. 59, 597-610. doi: 10.1080/10408398.2017.1384715

Chen, Y., Bystricky, P., Adeyeye, J., Panigrahi, P., Ali, A., Johnson, J. A., et al. (2007). The capsule polysaccharide structure and biogenesis for non-O1 Vibrio cholerae NRT36S: genes are embedded in the LPS region. BMC Microbiol. 7, 20-20. doi: 10.1186/1471-2180-7-20

Cheng, Z.-X., Chu, X., Wang, S.-N., Peng, X.-X., and Li, H. (2018). Six genes of ompA family shuffling for development of polyvalent vaccines against Vibrio alginolyticus and Edwardsiella tarda. Fish Shellfish Immunol. 75, 308-315. doi: 10.1016/j.fsi.2018.02.022

Cuesta, G., Suarez, N., Bessio, M. I., Ferreira, F., and Massaldi, H. (2003). Quantitative determination of pneumococcal capsular polysaccharide serotype 14 using a modification of phenol-sulfuric acid method. J. Microbiol. Methods 52, 69-73. doi: 10.1016/s0167-7012(02)00151-3

Dalsass, M., Brozzi, A., Medini, D., and Rappuoli, R. (2019). Comparison of opensource reverse vaccinology programs for bacterial vaccine antigen discovery. Front. Immunol. 10:113. doi: 10.3389/fimmu.2019.00113

Dong, H., Xiang, Q., Gu, Y., Wang, Z., Paterson, N. G., Stansfeld, P. J., et al. (2014). Structural basis for outer membrane lipopolysaccharide insertion. Nature 511, 52-56. doi: 10.1038/nature13464

Doytchinova, I. A., and Flower, D. R. (2007). VaxiJen: a server for prediction of protective antigens, tumour antigens and subunit vaccines. BMC Bioinf. 8:4. doi: 10.1186/1471-2105-8-4

Duperthuy, M., Binesse, J., Le Roux, F., Romestand, B., Caro, A., Got, P., et al. (2010). The major outer membrane protein OmpU of Vibrio splendidus contributes to host antimicrobial peptide resistance and is required for virulence in the oyster Crassostrea gigas. Environ. Microbiol. 12, 951-963. doi: 10.1111/j.1462-2920.2009.02138.x

Elmahdi, S., Dasilva, L. V., and Parveen, S. (2016). Antibiotic resistance of Vibrio parahaemolyticus and Vibrio vulnificus in various countries: a review. Food Microbiol. 57, 128-134. doi: 10.1016/j.fm.2016.02.008

Erik, L. L., Sonnhammer, Heijne, G. V., and Krogh, A. (1998). A hidden Markov model for predicting transmembrane helices in protein sequences. ISMB 6, $175-182$.

Fagerquist, C. K., and Zaragoza, W. J. (2018). Proteolytic surface-shaving and serotype-dependent expression of SPI-1 invasion proteins in Salmonella enterica subspecies enterica. Front. Nutr. 5:124. doi: 10.3389/fnut.2018.00124

Gao, D., Luan, Y., Liang, Q., and Qi, Q. (2016). Exploring the N-terminal role of a heterologous protein in secreting out of Escherichia coli. Biotechnol. Bioeng. 113, 2561-2567. doi: 10.1002/bit.26028

Garcia-Angulo, V. A., Kalita, A., Kalita, M., Lozano, L., and Torres, A. G. (2014). Comparative genomics and immunoinformatics approach for the identification of vaccine candidates for enterohemorrhagic Escherichia coli O157:H7. Infect. Immun. 82, 2016-2026. doi: 10.1128/iai.01437-13

Giuliani, M. M., Adu-Bobie, J., Comanducci, M., Aricò, B., Savino, S., Santini, L., et al. (2006). A universal vaccine for serogroup B meningococcus. PNAS 103, 10834-10839.

Guin, S., Saravanan, M., Chowdhury, G., Pazhani, G. P., Ramamurthy, T., and Das, S. C. (2019). Pathogenic Vibrio parahaemolyticus indiarrhoeal patients, fish and aquatic environments and their potential for inter-source transmission. Heliyon 5:e01743. doi: 10.1016/j.heliyon.2019.e01743

Hackbusch, S., Wichels, A., Gimenez, L., Döpke, H., and Gerdts, G. (2020). Potentially human pathogenic Vibrio spp. in a coastal transect: Occurrence and multiple virulence factors. Sci. Total Environ. 707:136113. doi: 10.1016/j. scitotenv.2019.136113
Han, L., Zheng, J., Wang, Y., Yang, X., Liu, Y., Sun, C., et al. (2016). Structure of the BAM complex and its implications for biogenesis of outer-membrane proteins. Nat. Struct. Mol. Biol. 23, 192-196. doi: 10.1038/nsmb.3181

He, Y., Xiang, Z., and Mobley, H. T. (2010). Vaxign: the first web-based vaccine design program for reverse vaccinology and an application for vaccine development. J. Biomed. Biotechnol. 2010:297505.

Hong, H., Patel, D. R., Tamm, L. K., and Van Den Berg, B. (2006). The outer membrane protein $\mathrm{OmpW}$ forms an eight-stranded $\beta$-barrel with a hydrophobic channel. J. Biol. Chem. 281, 7568-7577. doi: 10.1074/jbc. m512365200

Hornburg, D., Kruse, T., Anderl, F., Daschkin, C., Semper, R. P., Klar, K., et al. (2019). A mass spectrometry guided approach for the identification of novel vaccine candidates in Gram-negative pathogens. Sci. Rep. 9:17401.

Huang, Z., Tang, J., Li, M., Fu, Y., Dong, C., Zhong, J. F., et al. (2012). Immunological evaluation of Vibrio alginolyticus, Vibrio harveyi, Vibrio vulnificus and infectious spleen and kidney necrosis virus (ISKNV) combinedvaccine efficacy in Epinephelus coioides. Vet. Immunol. Immunopathol. 150, 61-68. doi: 10.1016/j.vetimm.2012.08.008

Jaiswal, V., Chanumolu, S. K., Gupta, A., Chauhan, R. S., and Rout, C. (2013). Jenner-predict server: prediction of protein vaccine candidates (PVCs) in bacteria based on host-pathogen interactions. BMC Bioinf. 14:211. doi: 10.1186/ 1471-2105-14-211

Kitamura, S., and Wolan, D. W. (2018). Probing substrate recognition of bacterial lipoprotein signal peptidase using FRET reporters(Letter). FEBS Lett. 592, 2289-2296. doi: 10.1002/1873-3468.13155

Koronakis, V., Sharff, A., Koronakis, E., Luisi, B., and Hughes, C. (2000). Crystal structure of the bacterial membrane protein TolC central to multidrug efflux and protein export. Nature 405, 914-919. doi: 10.1038/35016007

Köster, W. (2001). ABC transporter-mediated uptake of iron, siderophores, heme and vitamin B12. Res. Microbiol. 152, 291-301. doi: 10.1016/s0923-2508(01) 01200-1

Li, C., Ye, Z., Wen, L., Chen, R., Tian, L., Zhao, F., et al. (2014). Identification of a novel vaccine candidate by immunogenic screening of Vibrio parahaemolyticus outer membrane proteins. Vaccine 32, 6115-6121. doi: 10.1016/j.vaccine.2014. 08.077

Li, H., Xiong, X.-P., Peng, B., Xu, C.-X., Ye, M.-Z., Yang, T.-C., et al. (2009). Identification of broad cross-protective immunogens using heterogeneous antiserum-based immunoproteomic approach. J. Proteome Res. 8, 4342-4349. doi: $10.1021 /$ pr900439j

Li, H., Ye, M. Z., Peng, B., Wu, H. K., Xu, C. X., Xiong, X. P., et al. (2010a). Immunoproteomic identification of polyvalent vaccine candidates from Vibrio parahaemolyticus outer membrane proteins. J. Proteome Res. 9, 2573-2583. doi: 10.1021/pr1000219

Li, N., Yang, Z., Bai, J., Fu, X., Liu, L., Shi, C., et al. (2010b). A shared antigen among Vibrio species: Outer membrane protein-OmpK as a versatile vibriosis vaccine candidate in Orange-spotted grouper (Epinephelus coioides). Fish Shellfish Immunol. 28, 952-956. doi: 10.1016/j.fsi.2010.02.010

Mao, Z., Yu, L., You, Z., Wei, Y., and Liu, Y. (2007). Cloning, expression and immunogenicty analysis of five outer membrane proteins of Vibrio parahaemolyticus. Fish Shellfish Immunol. 23, 567-575. doi: 10.1016/j.fsi.2007. 01.004

Middleton, D. R., Paschall, A. V., Duke, J. A., and Avci, F. Y. (2018). Enzymatic hydrolysis of pneumococcal capsular polysaccharide renders the bacterium vulnerable to host defense. Infect. Immun. 86, 00316-00318e.

Naz, K., Naz, A., Ashraf, S. T., Rizwan, M., Ahmad, J., Baumbach, J., et al. (2019). PanRV: Pangenome-reverse vaccinology approach for identifications of potential vaccine candidates in microbial pangenome. BMC Bioinf. 20:123. doi: 10.1186/s12859-019-2713-9

Olaya-Abril, A., Jiménez-Munguía, I., Gómez-Gascón, L., and Rodríguez-Ortega, M. J. (2014). Surfomics: Shaving live organisms for a fast proteomic identification of surface proteins. J. Proteomics 97, 164-176. doi: 10.1016/j.jprot. 2013.03.035

Parsons, L. M., Lin, F., and Orban, J. (2006). Peptidoglycan recognition by Pal, an outer membrane lipoprotein. Biochemistry 45, 2122-2128. doi: 10.1021/ bi052227i

Pettis, G. S., and Mukerji, A. S. (2020). Structure, function, and regulation of the essential virulence factor capsular polysaccharide of Vibrio vulnificus. Int. J. Mol. Sci. 21:3259. doi: 10.3390/ijms21093259 
Pizza, M., Scarlato, V., Masignani, V., Giuliani, M. M., Aric, B., Comanducci, M., et al. (2000). Identification of vaccine candidates against serogroup B meningococcus by whole-genome sequencing. Science 287, 1816-1820. doi: 10.1126/science.287.5459.1816

Pore, D., and Chakrabarti, M. K. (2013). Outer membrane protein A (OmpA) from Shigella flexneri 2a: A promising subunit vaccine candidate. Vaccine 31, 3644-3650. doi: 10.1016/j.vaccine.2013.05.100

Rehman, T., Yin, L., Latif, M. B., Chen, J., Wang, K., Geng, Y., et al. (2019). Adhesive mechanism of different Salmonella fimbrial adhesins. Microb. Pathog. 137:103748. doi: 10.1016/j.micpath.2019.103748

Rodríguez-Ortega, M. J. (2018). “"Shaving” live bacterial cells with proteases for proteomic analysis of surface proteins," in The Surfaceome: Methods and Protocols, eds K. R. Boheler and R. L. Gundry (New York, NY: Springer), 21-29. doi: 10.1007/978-1-4939-7553-2_2

Rodríguez-Ortega, M. J., Norais, N., Bensi, G., Liberatori, S., Capo, S., Mora, M., et al. (2006). Characterization and identification of vaccine candidate proteins through analysis of the group A Streptococcus surface proteome. Nat. Biotechnol. 24, 191-197. doi: 10.1038/nbt1179

Rollauer, S. E., Sooreshjani, M. A., Noinaj, N., and Buchanan, S. K. (2015). Outer membrane protein biogenesis in Gram-negative bacteria. Philos. Trans. R. Soc. Lond. B. Biol. Sci. 370:20150023.

Sanober, G., Ahmad, S., and Azam, S. S. (2017). Identification of plausible drug targets by investigating the druggable genome of MDR Staphylococcus epidermidis. Gene Rep. 7, 147-153. doi: 10.1016/j.genrep.2017.04.008

Schlingmann, B., Castiglia, K. R., Stobart, C. C., and Moore, M. L. (2018). Polyvalent vaccines: High-maintenance heroes. PLoS Pathog. 14:e1006904. doi: 10.1371/journal.ppat.1006904

Serruto, D., Adu-Bobie, J., Capecchi, B., Rappuoli, R., Pizza, M., and Masignani, V. (2004). Biotechnology and vaccines: application of functional genomics to Neisseria meningitidis and other bacterial pathogens. J. Biotechnol. 113, 15-32. doi: 10.1016/j.jbiotec.2004.03.024

Shinoy, M., Dennehy, R., Coleman, L., Carberry, S., Schaffer, K., Callaghan, M., et al. (2013). Immunoproteomic analysis of proteins expressed by two related pathogens, Burkholderia multivorans and Burkholderia cenocepacia, during Human Infection. PLoS One 8:e80796. doi: 10.1371/journal.pone.0080796

Singh, R., Capalash, N., and Sharma, P. (2017). Immunoprotective potential of BamA, the outer membrane protein assembly factor, against MDR Acinetobacter baumannii. Sci. Rep. 7:12411.

Smith, S. G. J., Mahon, V., Lambert, M. A., and Fagan, R. P. (2007). A molecular Swiss army knife: OmpA structure, function and expression. FEMS Microbiol. Lett. 273, 1-11. doi: 10.1111/j.1574-6968.2007.00778.x

Solis, N., and Cordwell, S. J. (2011). Current methodologies for proteomics of bacterial surface-exposed and cell envelope proteins. Proteomics 11, 3169-3189. doi: 10.1002/pmic.201000808

The UniProt (2018). UniProt: a worldwide hub of protein knowledge. Nucl. Acids Res. 47, D506-D515.

Tobes, R., and Ramos, J. L. (2002). AraC-XylS database: a family of positive transcriptional regulators in bacteria. Nucl. Acids Res. 30, 318-321. doi: 10.1093/ nar/30.1.318

Tsirigotaki, A., De Geyter, J., Šoštarić, N., Economou, A., and Karamanou, S. (2017). Protein export through the bacterial Sec pathway. Nat. Rev. Microbiol. 15, 21-36. doi: 10.1038/nrmicro.2016.161

Turner, J. W., Tallman, J. J., Macias, A., Pinnell, L. J., Elledge, N. C., Nasr Azadani, D., et al. (2018). Comparative genomic analysis of Vibrio diabolicus and six taxonomic synonyms: A first look at the distribution and diversity of the expanded species. Front. Microbiol. 15:1893. doi: 10.3389/fmicb.2018.01893

Tusnády, G. E., and Simon, I. (2001). The HMMTOP transmembrane topology prediction server. Bioinformatics 17, 849-850. doi: 10.1093/bioinformatics/17. 9.849

Wang, C., Liu, Y., Li, H., Xu, W.-J., Zhang, H., and Peng, X.-X. (2012). Identification of plasma-responsive outer membrane proteins and their vaccine potential in Edwardsiella tarda using proteomic approach. J. Proteomics 75, 1263-1275. doi: 10.1016/j.jprot.2011.11.001

Wang, Q., Ji, W., and Xu, Z. (2020). Current use and development of fish vaccines in China. Fish Shellfish Immunol. 96, 223-234. doi: 10.1016/j.fsi.2019.12.010

Wilson, M. M., and Bernstein, H. D. (2016). Surface-exposed lipoproteins: An emerging secretion phenomenon in Gram-negative bacteria. Trends Microbiol. 24, 198-208. doi: 10.1016/j.tim.2015.11.006

Wimley, W. C. (2003). The versatile $\beta$-barrel membrane protein. Curr. Opin. Struct. Biol. 13, 404-411. doi: 10.1016/s0959-440x(03)00099-x

Wizemann, T. M., Adamou, J. E., and Langermann, S. (1999). Adhesins as targets for vaccine development. Emerg. Infect. Dis. 5, 395-403. doi: 10.3201/eid0503. 990310

Xu, C., Kuang, H., and Xu, L. (2019). "Pathogen Immunoassay in Food," in Food Immunoassay, eds H. Kuang, C. Xu, and L. Xu (Singapore: Springer), 255-319. doi: 10.1007/978-981-13-9034-0_8

$\mathrm{Xu}$, J., and Zhang, Y. (2010). How significant is a protein structure similarity with TM-score $=0.5$ ? Bioinformatics $26,889-895$. doi: $10.1093 /$ bioinformatics/ btq066

Yang, J., Anishchenko, I., Park, H., Peng, Z., Ovchinnikov, S., and Baker, D. (2020). Improved protein structure prediction using predicted interresidue orientations. PNAS 117, 1496-1503. doi: 10.1073/pnas.1914677117

Yang, J., Yan, R., Roy, A., Xu, D., Poisson, J., and Zhang, Y. (2015). The I-TASSER Suite: protein structure and function prediction. Nat. Methods 12, 7-8. doi: 10.1038/nmeth.3213

Yonekita, T., Morishita, N., Arakawa, E., and Matsumoto, T. (2020). Development of a monoclonal antibody for specific detection of Vibrio parahaemolyticus and analysis of its antigen. J. Microbiol. Methods 173:105919. doi: 10.1016/j.mimet. 2020.105919

Yu, C., Lin, C., and Hwang, J. (2004). Predicting subcellular localization of proteins for Gram-negative bacteria by support vector machines based on n-peptide compositions. Protein Sci. 13, 1402-1406. doi: 10.1110/ps.03479604

Yu, N. Y., Wagner, J. R., Laird, M. R., Melli, G., Rey, S., Lo, R., et al. (2010). PSORTb 3.0: Improved protein subcellular localization prediction with refined localization subcategories and predictive capabilities for all prokaryotes. Bioinformatics 26, 1608-1615. doi: 10.1093/bioinformatics/btq249

Yu, Q. Q., Niu, M. Y., Yu, M. Q., Liu, Y. H., Wang, D. P., and Shi, X. M. (2016). Prevalence and antimicrobial susceptibility of Vibrio parahaemolyticus isolated from retail shellfish in Shanghai. Food Control 60, 263-268. doi: 10.1016/j. foodcont.2015.08.005

Zeng, L., Wang, D., Hu, N., Zhu, Q., Chen, K., Dong, K., et al. (2017). A novel Pangenome reverse vaccinology approach employing a negative-selection strategy for screening surface-exposed antigens against leptospirosis. Front. Microbiol. 14:396. doi: 10.3389/fmicb.2017.00396

Zgurskaya, H. I., Krishnamoorthy, G., Ntreh, A., and Lu, S. (2011). Mechanism and function of the outer membrane channel TolC in multidrug resistance and physiology of enterobacteria. Front. Microbiol. 2:189. doi: 10.3389/fmicb.2011. 00189

Zha, Z., Li, C., Li, W., Ye, Z., and Pan, J. (2016). LptD is a promising vaccine antigen and potential immunotherapeutic target for protection against Vibrio species infection. Sci. Rep. 6:38577.

Conflict of Interest: The authors declare that the research was conducted in the absence of any commercial or financial relationships that could be construed as a potential conflict of interest.

Copyright (C) 2021 Wang, Liu, Guo, Liu, Yuan, Guo and Pan. This is an open-access article distributed under the terms of the Creative Commons Attribution License (CC BY). The use, distribution or reproduction in other forums is permitted, provided the original author(s) and the copyright owner(s) are credited and that the original publication in this journal is cited, in accordance with accepted academic practice. No use, distribution or reproduction is permitted which does not comply with these terms. 